\title{
Internet of Things and Wireless Sensor Network for Smart Cities
}

\author{
Raj Mondal ${ }^{1}$, Tufail Zulfi ${ }^{2}$ \\ 1 Assistant Professor, Department of Computer Science \& Engineering, Maulana Azad National Urdu University, \\ Hyderabad-500032, India \\ 2 Assistant Professor, Department of Electronics \& Communication Engineering, Maulana Azad National Urdu University, \\ Hyderabad-500032, India
}

\begin{abstract}
As the Internet of Things (IoT) and the Wireless Sensor Network (WSN) are becoming a reality, their interconnections for smart devices are increasing. Smart devices are integrated with sensors and embedded system to offer advanced services, which combined with the IoT to developing a smart city. The Internet of Things shall be able to connect the physical objects through sensors, actuator networks and then control them. It describes the most significant work performed in the area of WSN combined with the IoT for creating a smart city. This paper also focus specifically to an urban IoT system that shows a broad category, are characterized by their specific application domain. Sensing, actuating and controlling for developing an urban area using IoTs are the most advanced communication technologies to support services for smart city and for citizens. This paper also provides a comprehensive survey of smart city concept, technologies, various challenges and architecture.
\end{abstract}

Keywords: Internet of Things (IoT), identifications and tracking, Wireless Sensor Networks (WSN), sensors, actuators, Radio Frequency Identifications(RFID).

\section{Introduction}

The Sensor networks and the Internet of Things (IoT) is a recent communication paradigm for digital world that will increase in near future, in which the objects (electronic equipment) of everyday life will be embedded with sensors, actuators, microcontroller also AI for digital communication and several networking protocols that will make them able to communicate with one another and with the users, becoming an integral part of the digitization. The WSN concept, aims at making the Internet even more immersive and easy accessible. Hence, by enabling easy access and interaction with a wide variety of devices such as home appliances, surveillance cameras, humidity sensors, actuators, forest fire detection sensors, vehicles tracker, mobiles, and cars and also many objects surrounding us will be connected into networks in one form or another. RF identification (RFID), sensor technology, and other smart technologies will be embedded into a variety of applications, the IoT will monitor those devices through sensors for development of a number of applications that make use of the potentially large amount and variety of data generated by such objects to provide new smart services to citizens, companies and also public administrations. This IoT paradigm indeed finds application in different domain, such as home automation, industrial automation, medical aids, mobile healthcare, elderly assistance, intelligent energy management and smart grids automotive, traffic management, and many others [1].

A smart city is one that uses internet of things to make the critical infrastructure components and services of a city administration, education, healthcare, public safety, real estate, transportation and utilities more aware, interactive and efficient. The application of the IoT paradigm to an urban area is of particular interest, as it responds to the strong push of many national governments to use ICT solutions in the management of public affairs which is Smart City concept [2]. In [9], it is shows that the revolution will be possess by integrated of electronics into physical objects, creating them smart and it also integrated with the physical word that will gives a new opportunity for ICT sector.

The main aim of smart city is to make a better utilize of the public resources, increasing the quality of the services provides to the citizens, while reducing the operational costs of the public administrations. While this objective cannot be achieved with technology or ICT alone, leveraging the deployment of IoT, ICT \& Big data within a city can go a long way to reaching this goal. In a survey we know that by 2050, seventy percent of the global 
population will live in urban areas like smart cities. Fortunately, the digital revolution holds great promise for responding to many of the challenges created by imminent urbanization. This review paper also gives a pictorial view of the current state of the creative activity on the Internet of Things (IoT). The future development and potential of IoT has been estimated by using some technologies such as networking, cloud computing, Big Data, robotics, sensor network etc. Many telecom and software industries like HP, Cisco, and Ericsson have been working on the development or evolution of IoT embedded with smart city.

An urban IoT, have many facilities that may bring a number of benefits such as management and optimization of traditional public services, like transport and parking facilities, lighting, surveillance and maintenance of public areas, preservation of cultural heritage, garbage collection, public health care i.e. hospitals and school. Therefore, the availability of different types of data that will store in the cloud or collected by a data warehouse of urban IoT, it should increase the transparency and promote the actions of the local government or municipality toward the citizens, that increase the awareness of people about the status of their city, their life style. Therefore, the IoT paradigm applications to the Smart City is attractive and expansive to local government and administrations, thus it take times for adopting the IoT technology in a wide manner. The aim of this paper is to describe a framework for smart city design in an urban IoT. Here we discuss some necessary characteristic of an urban IoT area and their services provided by the local governments. Then this paper also shows some web-based applications, sensor protocols, network technologies, Layers and security for Smart City development.

\section{Smart City Concept and Services}

The concept of smart city is basically the proper utilization of public resources, maximize the Quality of Services that provides to its citizens while minimizing the operational costs. While this aim cannot be accomplished with technology alone, also increasing the deployment of IoT within an urban area can go a broad way to achieve this goal. IoT, Big Data and Sensors are expected to develop the utilization of networking technology in an urban centers and controlling them. In a survey we know that today nearly three hundred million machine-to-machine sim card in use an ever increasing number of connected smart devices. Domains that shows the lowest to highest potential economic impact as shown below:

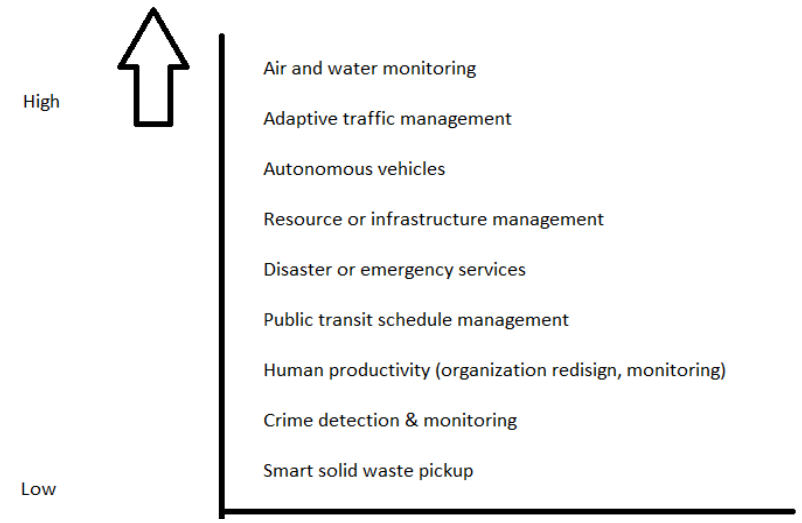

Fig. 1: Potential economic impact

The above figure- 1 depicted that according to McKinsey Global Institute survey the potential direct economic impact increases of $\$ 930$ billion to $\$ 1.7$ trillion per year by 2025. Basically Smart city development plans are categorized into three stages [4]: a) initial infrastructure construction stage b) data-processing facility construction stage and c) end-phase service platform construction stage. A huge number of smart city projects gives large opportunities to telecom industry, original equipment manufacturer (OEM), systems integration enterprises, data aggregation and analysis or service enterprises and also different telecom operators.

In the USA, the National Intelligence Council (NIC) predicts that by 2025 Internet \& sensor nodes may occupy in our everyday life. Many developed countries like USA, Europe and some countries of Asia like India, china and japan, they are now a days considering internet of things (IoT) as an area of innovation and growth. Therefore, the development of smart city using Internet of Things (IoT) and their research is going on in various university, some telecom \& software industries and many research organization across the world.

Several cities are now focused to enlarge the use of IoT for smart city development to improve services such as water management quality, waste management, energy consumption etc. The following services are needed for the smart city development.

\section{1 Structural Health of Buildings}

Exact maintenance needed for the old historical buildings of a city, continuous monitoring and identification of those buildings through wireless sensors. Buildings conditions, structural integrity measurements, such as vibration or building stress can be monitored by the sensor nodes located in the historical buildings. The pollution level can be monitored by the atmospheric sensors in the 
surrounding areas, temperature $\&$ humidity sensors and also vibrator sensor for earthquake alerts are located in the buildings. Therefore the wireless sensor nodes can organize or collected those data and send to the base station for proper monitoring using some routing algorithms such as directed diffusion, energy efficient and cluster based routing. These environmental conditions [5] can be monitored by the use of IoT. This database are stored by cloud computing technology. If the impact of light earthquake or fire detection or anything happen in the building, immediate detection will be monitored by the sensors located in the building for the protection and to make the citizens aware of the take care in the city historical buildings. However this IoT services required the installation of wireless sensor nodes in building and surrounding areas and to control them which needed more investment by the local or regional government.

\section{2 Waste Management}

Waste management is a process to manage waste from its inception to final disposal and also recycling them. This not only include waste but also transport, treatment and disposal of waste with regulation and proper monitoring. According to different countries and regions waste collection methods are different. For industrial or commercial waste collection services are provided by private companies whereas domestic waste collection services are by the local government authority. Most of the European countries uses curbside waste segregation method of disposal in which waste are collected in a regular intervals by specialized trucks. Most of the urban area have lots of waste disposal problems because of cost of the services and storage of garbage. Today ICT solves some this problems. The use of intelligent waste container that detect the level of garbage loads and proper optimization of the collector trucks route, that can decrease the cost of waste collection and within time period recycling[6] is done. In that sense IoT shall connect the sensor in the collector trucks for controlling the garbage load collector and also uses of optimization software process for recycling the waste.

\section{3 Environmental Air Quality}

Everyone knows that the greenhouse gases are increases in day to day life. The IoT can take care of those gases that means it reduces the greenhouse gas emission, it creates \& maintains more amount of solar energy or renewable energy rather than energy coming from coal, gas or oil. Because the outcomes of burning a coal, gas or oil is more harmful for the natural air. So it can monitor the air quality in a crowded area, or in long traffic, parks or in locality area. The realization of such services needed air quality and pollution sensors deployed all over the urban area. This sensor data are controlled and made publicly available to citizens for their healthiest life.

\section{4 Noise Monitoring}

One major issue is sound pollution or acoustic pollution in the city center, already the government have issued a specific law for the noise pollution in city area at specific hours. The urban smart city IoT system can offer noise monitoring services [7] to measure the amount of noise in public area or in a traffic or in a locality. Some sound detection algorithm have been developed and used in noise monitoring system, this service can reduce the acoustic pollution at the nights in the city. Also the installation of acoustic sensor or sound detectors are widely deployed in a smart city region.

\section{5 Traffic Congestion}

This application can get traffic information of roads such as road traffic conditions and congested locations by tracking the location information of a huge number of vehicles. Thus the system will give a new information to the driver to choose the most efficient route for traffic congestion. The IoT system can get road traffic information or traffic congestion by different types of tracker like GPS, RFID (Radio Frequency Identification) or sensor based camera. Also sensor based camera that monitor traffic conditions and detect speed of the vehicles are also deployed in many cities. Controlling the traffic conditions are more easy using wireless sensors and GPS based modern vehicles in most of the urban area.

\section{6 City Energy Consumption}

The urban IoT system may also provide a service to monitor the energy consumption of the entire city, which gives a detail view to the authority and citizens. Proper monitoring the amount of energy uses in different activity like house hold lighting, transportation, street lights, traffic camera, heating or cooling of building or office and so on. It is possible to identify the sources of energy consumption and to set priorities in order to proper utilization. Most of the smart city area needed power draw monitoring devices must be embedded with power grid that's result to enhance these service with active functionalities to control local power production structures. 


\section{General Architecture of IoT}

The general architectures of IoT systems are domainspecific or application specific solutions. We have to integrate multiple functions and resources into a large system than can reduce the total IoT cost. Thus we need to design the IoT architecture with a layer specific and generic with open interfaces, resources and current technologies [10]. During architectural development of IoT we have to focus on the total cost and time for devices, deployment \& development. The General architecture of IoT are as follows:

\section{1 Sensing Layer}

This layer is the interconnection of sensors, controller, RFID readers and GPS to IoT network layer. This layer also called sensing and gateway platform. Different types of hardware, software interface and data formats are proposed for IoT terminal, IoT gateway and IoT tip nodes. This IoT terminal, gateway and tip nodes can consists of flexible module, control module, common interface module and communication module. The common interface module can organise physical interfaces of different sensor nodes. And common control module can connect GPS, RFID readers, controller and sensors with a specific protocol. Different application and the software interfaces of an IoT terminal and IoT gateway are needed to be self-configure and self-adapt.

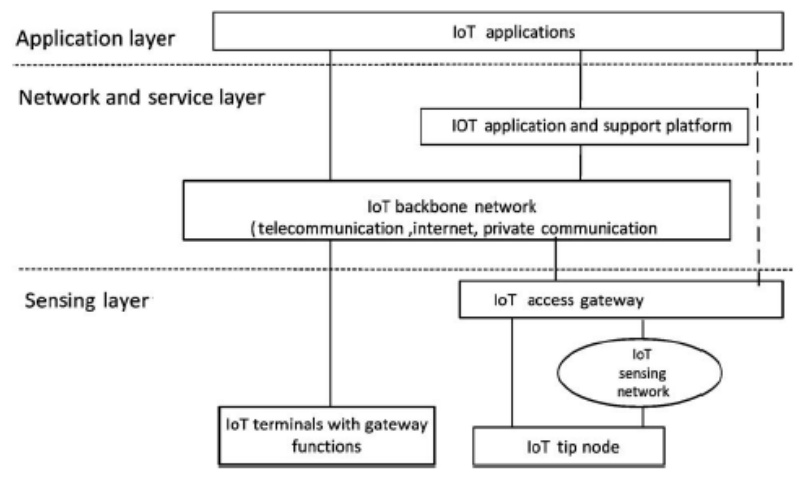

Fig. 2: General Architecture of IoT

\section{2 Network \& Service Layer}

Network layer also called Resource and Administration Platform. It includes backbone network which consists of 3G, 4G internet, Optical fibre network, Ethernet network, satellite network or any other private network. The resource and administration platform operates by different
IoT applications which include data storage, data processing and security management. This network layer also provides different control functions such as authorize access and transport resource control function, mobility management, encryption or authentication, authorization for IoT.

\section{3 Application Layer}

This application platform provides some interfaces or common functions and also application programming interface which will be capable of accessing different devices with a common language protocol or procedures. Many applications can be developed using IoT application provider and API. Users can get various applications information and subscribe these applications through application layer IoT.

\section{Applications of IoT}

The IoT applications have been developed in many fields such as: Industry application, smart agriculture, intelligent transport, smart grid, smart environmental protection, smart safety, smart building, smart medical etc. We have to discuss here few of its application such as:

\section{1 Smart Building}

It improve the house electricity usage means electric energy saving with uses of motion sensor lights which can dim or shut off the light when a room is empty. Smart house will also give alarm signal when there is any leakage of gas or smoke of any fire using such kind of sensors. Uses of electric energy monitoring when it will reaches a specific threshold using smart meter.

\section{2 Smart Water}

Most of the city's critical infrastructure is its water supply. With populations in urban area are growing as well as water consumption will also grow. The term Smart Water means the proper utilization of fresh water and waste water, its infrastructure resources and the transport i.e. supply and management cost effectively. City's water supply and management have to strong and prevent water waste using data from detecting water pressure and leaks in the long term to maintain its growth. 


\section{3 Smart Lighting}

Energy consumption of city's street lights is an important feature. The street light service must be depend on the time of the day, weather condition and the presence of people, it also include light sensor in order to proper utilization of energy, such a service needs to improve for smart city development. It is also possible to detect the faults for every damaged street light using street light controller. If we use solar street light, then it save fuel cost or other energy cost from power grid.

\section{4 Smart Parking}

The smart parking service is based on traffic sensors and intelligent display that select the route along the best path for parking in the city [8]. Its benefits are take less time to locate a parking spot as a result fewer $\mathrm{CO}$ emission from the vehicles, less traffic congestion and happier and healthier way of life. Smart parking in many urban area depends on the investment by the municipality of that area. Many urban residents, mobility development is the main area. It should be lower cost of transportation and reduce time for transportation, avoid traffic jams and follow another shorter route, should have ample parking. Public complaints about commute times or availability of parking spot in smart city area district. The vehicles parking sector in others country like USA or Europe is rapidly innovating with the smart systems. It also include advance automation system and software management for parking spot booking. It also uses rapid development of wireless technology.

\section{5 Smart Medical}

It is involved the use of ICT (Information \& Communication Technology) to help patients and doctors about the health diagnosis and challenges faced by the patients. This smart medical technology include both the software as well as hardware services like telemedicine, web based analysis, e-Health, e-Patient, m-Health and clinic or remote monitoring sensor technology. Smart medical is a multi-disciplinary field, basically it is called digital health. Many researcher, scientists, clinicians with a wide range of expertise are involved in this domain.

\section{6 Smart Environmental Protection}

It focuses on the domain of pollution source monitoring, water quality monitoring and air quality monitoring. These three domain's data are collected by this technology and proper monitoring. This technology also prevent and control degradation of land, water, vegetation and air, raise awareness and understanding of the environmental development, ensure sustainable and equitable use of resources without degrading the environmental factors. Also promote international cooperation.

\section{Conclusion and Future Work}

The Internet of Things has rapidly grow according to our lifestyle. For example such as moving interactions between the citizens and the physical world with virtually connected through the everyday use of internet communications technology and also the IoT has a potential to add a new logic to this process by enabling the wireless sensor network and its different protocols. This review paper also gives a pictorial view of the current state of the creative activity on the Internet of Things (IoT). The future development and potential of IoT has been estimated by using some technologies such as networking, cloud computing, Big Data, robotics, sensor network etc. The main aim of smart city is to make a better utilize of the public resources, increasing the quality of the services provides to the citizens, while reducing the operational costs of the public administrations. Therefore in this review paper we have discussed the most basic, important and fundamental aspects of Internet of Things technology which will be done in near future and also discussed some applications of IoT and its services that will help for any of the smart city development. So we conclude that the use of wireless sensors network, RFID and ICT have been leading for the smart city development in an urban area which is a great invention of this IoT technology.

In this report we conceptually proposed the three layer architecture for smart city IoT. In future endeavors we have plan to carry out a simulated experiment that will collect data and monitoring different sector with the help of sensors and other ICT for development of urban area. This simulated experiment will contains the accuracy and efficiency of the proposed framework. Therefore to evaluate the standardization and generalizability of the proposed model, in order to develop the smart city architecture.

\section{Acknowledgments}

The authors gratefully acknowledge the support from Department of Computer Science \& Engineering, University of Kalyani, West Bengal, India. 


\section{References}

[1] P. Bellavista, G. Cardone, A. Corradi, and L. Foschini, "Convergence of MANET and WSN in IoT urban scenarios," IEEE Sens. J., vol. 13, no. 10, pp. 3558-3567, Oct. 2013.

[2] H. Schaffers, N. Komninos, M. Pallot, B. Trousse, M. Nilsson, and A. Oliveira, "Smart cities and the future internet: Towards cooperation frameworks for open innovation," The Future Internet, Lect.NotesComput. Sci., vol. 6656, pp. 431-446, 2011.

[3] D. Cuff, M. Hansen, and J. Kang, "Urban sensing: Out of the woods," Commun. ACM, vol. 51, no. 3, pp. 24-33, Mar. 2008.

[4] S. Chen, H.Xu, D. Liu, and Bo Hu, "A Vision of IoT: Applications, Challenges, and Opportunities with China Perspective," IEEE Internet of Things Journal, Vol. 1, No. 4, pp.349-359, August 2014.

[5] J. P. Lynch and J. L. Kenneth, "A summary review of wireless sensors and sensor networks for structural health monitoring," Shock and Vibration Digest, vol. 38, no. 2, pp. 91130, 2006.

[6] T.Nuortio, J.Kytöjoki, H.Niska, and O.Bräysy, "Improved route planning and scheduling of waste collection and transport," Expert Syst. Appl., vol. 30, no. 2, pp. 223-232, Feb. 2006.

[7] N. Maisonneuve, M. Stevens, M. E. Niessen, P. Hanappe, and L. Steels, "Citizen noise pollution monitoring," in Proc. $10^{\text {th }}$ Annu. Int. Conf. Digital Gov.Res.: Soc.Netw.:Making Connec. Between Citizens, DataGov., 2009, pp. 96-103.

[8] S. Lee, D. Yoon, and A. Ghosh, "Intelligent parking lot application using wireless sensor networks," in Proc. Int. Symp. Collab. Technol. Syst., Chicago, May 19-23, 2008, pp. 48-57.

[9] Daniele Miorandi, Sabrina Sicari et.al., "Internet of ThingsVision, applications and research challenges", Ad Hoc Networks, vol. 10, 2012, pp.1497-1516.

[10] J. Gubbi et al., "IoT: A vision, architectural elements, and future directions," Future Gener. Comput. Syst., vol. 29, no. 7, pp. 1645-1660, Sep. 2013.

[11] J. P. Lynch and J. L. Kenneth, "A summary review of wireless sensors and sensor networks for structural health monitoring," Shock and Vibration Digest, vol. 38, no. 2, pp. 91130, 2006.

[12] A. R. Al-Ali, I. Zualkernan, and F. Aloul, "A mobile GPRSsensors array for air pollution monitoring," IEEE Sensors ,vol.10,no.10,pp.1666-1671, Oct. 2010.

[13] D. Guinard, V. Trifa, F. Mattern, E. Wilde, "From the Internet of Things to the Web of Things: Resource Oriented Architecture and Best Practices", Springer, New York, Dordrecht, Heidelberg, London, 2011.

[14] A. Jules, "RFID security and privacy: a research survey", IEEE Journal on Selected Areas in Communications, vol. 24, no. 2, pp. 381-394, 2006.

Raj Kumar Mondal is an Assistant Professor in Department of Computer Science \& Engineering, Maulana Azad National Urdu University, Hyderabad, India. He has received his M.Tech in CSE in the year 2012 from West Bengal University of Technology, West Bengal, India. After M.Tech he was joined as an Assistant Professor in Department of Computer Science \& Engineering, Hooghly Engineering \& Technology College, West Bengal, India. $\mathrm{He}$ is a life member of Indian Science Congress Association. His area of interests are Wireless Sensor Network, Internet of Things, Ad-hoc Network and Network Security.

Tufail Ahmad Zulfi is an Assistant Professor in Department of Electronics \& Communication Engineering, Maulana Azad National Urdu University, Hyderabad, India. He has received his M.Tech in Electronics \& Communication Engineering in the year 2013 from MANIT, Bhopal, India. Before M.Tech he was joined as an Assistant Professor in Department of Electronics \& Communication Engineering, MACET, Bihar, India. He is a life member of Indian Science Congress Association. His area of interests are wireless communication, cognitive radio, WSN, VLSI design and microwave. 